\title{
Schichtarbeitende im Dauer-Jetlag
}

\author{
Interview Im Wechsel je eine Woche in Tokio, Berlin und Denver \\ leben - und das jahrelang? So kann sich für Pflegefachkräfte der \\ wöchentliche Wechsel von Früh-, Spät- und Nachtschicht anfühlen, \\ sagt Dr. Hans-Günter Weeß, Leiter des interdisziplinären Schlafzen- \\ trums im Pfalzklinikum und Vorstandsmitglied der Deutschen Gesell- \\ schaft für Schlafforschung und Schlafmedizin. In Seminaren verhilft er \\ Angehörigen von Gesundheitsberufen zu gesundem Schlaf.
}

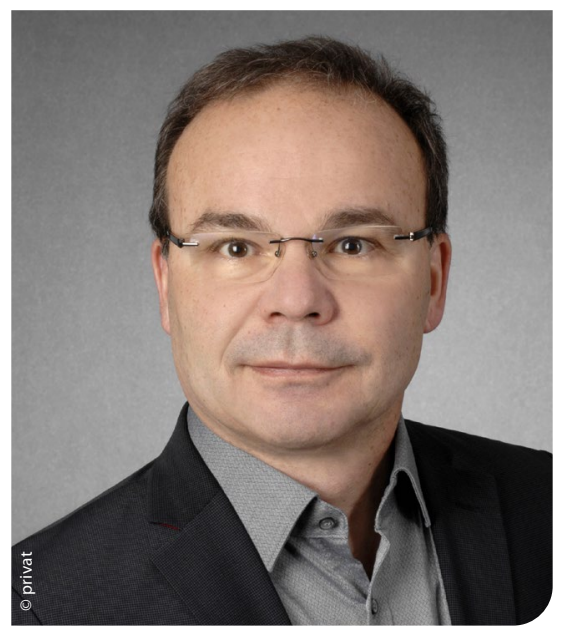

krankungen bis hin zur Depression. Die Dauermüdigkeit erhöht nachweislich auch das Risiko von Verkehrsunfällen oder Fehlern im Dienst. kommen mit der Spät- oder auch der Nachschicht besser zurecht. Für sie fühlt sich eher die Frühschicht an wie eine halbe Nachtschicht. Wenn dieselbe Schicht mehr als fünf bis sieben Tage andauert, beginnt unsere innere Uhr, sich umzustellen. Kommen ein wöchentlicher Wechsel und eine Rückwärtsrotation der Schicht hinzu, kann man das mit einem ständigen Ortswechsel zwischen drei Zeitzonen vergleichen. Stimmen die innere und die äußere Uhr nicht überein, sprechen wir von einem sozialen Jetlag. Auch ein direkter Wechsel von der Spät- in die Fühschicht ohne Pausentag ist problematisch. Beträgt die Ruhe- oder Schlafzeit weniger als sechs Stunden, ist Übermüdung programmiert.
_Haben Pflegefachkräfte andere Schlaf-
probleme als beispielsweise Schichtarbei-
tende in der Industrie?
Weeß: Im Rahmen unserer Seminare beob-
achten wir, dass Pflegefachkräfte es mit be-
sonderen Belastungen zu tun haben: Der
Schichtplan wird wegen Krankheitsausfällen
geändert, Fachkräfte springen füreinander ein
oder bleiben länger, wenn Unvorhergesehenes
passiert. Eine Sonderregelung erlaubt Kran-
kenhäusern die ungünstige Rückwärts-
Schichtrotation, was in der Wirtschaft nicht
zulässig ist. Dazu kommen die körperliche und
psychische Belastung sowie die Tatsache, dass che oder leistungsbezogene Einschränkungen am Tag, selbst an schichtfreien Tagen oder im Urlaub. Dies betrifft etwa 20\% der Schichtarbeitenden.

\footnotetext{
_Welche Schichten verursachen die meisten Probleme und spielt die Abfolge der Schichtdienste für die Entstehung von Schlafstörungen eine Rolle?

Weeß: Die Nachtschicht ist für die Meisten am schwierigsten, sie widerspricht bekanntlich unserem Biorhythmus. Man muss wach bleiben, wenn man müde ist und schlafen, wenn es hell und das Umfeld aktiv ist. Es hängt vom Chronotypen ab, wer welche Schicht besser verträgt: Frühaufsteher, wir nennen sie Lerchen, quälen sich oft durch die
}

\section{- Schichtarbeitende setzen sich enorm unter Druck, ordentlich zu schlafen und sich zu erholen.}

viele Fachkräfte sich auch zuhause mit den Schicksalen der Patienten beschäftigen. Daher schätze ich den Anteil der Insomnien im Gesundheitswesen höher ein als in der freien Wirtschaft.

\footnotetext{
_ Warum ist es so schwer, nach einer anstrengenden Schicht und trotz Übermüdung gut zu schlafen?

Weeß: Neben Familie oder Haushalt bleibt kaum noch Zeit für bewusste Entspannung. Diese ist aber wichtig zur Regeneration von
} 
Körper und Psyche sowie für guten Schlaf. Schichtarbeitende, das zeigen unsere Studien, neigen häufiger zu nächtlichen Grübeleien und setzen sich enorm unter Druck, ordentlich zu schlafen und sich zu erholen. Sie befürchten, nicht schnell genug einzuschlafen oder den Wecker zur Frühschicht zu überhören. Diese Anspannung und die intensive gedankliche Beschäftigung mit dem eigenen Schlafverhalten begünstigen die Schlafprobleme umso mehr. Wer unbedingt schlafen will, bleibt wach.
_Wie gehen Sie bei einer Therapie üblicherweise vor?

Weeß: Am Anfang steht eine Analyse des individuellen Schlafverhaltens, etwa anhand eines Schlafprotokolls. Nach der Diagnose werden die genannten Techniken nach einem persönlichen Plan zusammengestellt und über Wochen hinweg eingeübt. Die meisten Menschen schaffen es aus eigener Kraft, ihr Schlafverhalten und ihre psychische Einstellung zum Schlaf zu ändern.

\section{- Wir beobachten bei den Arbeitgebern ein steigendes Interesse an Seminaren zum Schlaf - das Thema wird wichtiger.}

\begin{abstract}
_ Das klingt so, als wäre guter Schlaf vor allem Kopfsache - wie kann Menschen mit Schlafstörungen professionell geholfen werden?

Weeß: Schlafprobleme können verschiedene Ursachen haben, neben äußeren Faktoren wie dem Schichtdienst spielen auch Gesundheit, Familiengeschichte und Persönlichkeit eine Rolle. Nach unserer Erfahrung werden chronische Insomnien aber vor allem durch falsche Verhaltensweisen und störende Gedanken verursacht. Das Gute daran ist, dass man diese mit kognitiv-verhaltenstherapeutischen Maßnahmen gut in den Griff bekommt. Eine entspannte Haltung zum Schlaf ist erlernbar. Wir arbeiten vor allem mit einer Kombination aus Stressmanagement, körperlichen und psychischen Entspannungstechniken sowie Maßnahmen der Schlafhygiene. So nennen wir bestimmte Verhaltensregeln. Es geht darum, einen Schlafrhythmus zu finden, Schlafdruck aufzubauen und die Grübeleien im Bett zu beenden.
\end{abstract}

\footnotetext{
- Was bedeutet "Schlafdruck aufbauen"? Weeß: Es geht darum, die biologischen und psychologischen Bedingungen für einen guten Schlaf zu entwickeln: Man muss ausreichend lange wach sein, damit sich der Schlafbotenstoff Adenosin extrazellulär im Gehirn ansammeln kann. Weiterhin sollte es für den Schlafbotenstoff Melatonin ausreichend dunkel sein. Auf der psychischen Seite benötigen wir optimale Entspannung, so dass die biologischen Faktoren wirken und Schlaf auftreten kann.
}

— Was können von Insomnie Betroffene selbst tun, um ihre Schlafprobleme in den Griff zu bekommen?

Weeß: Manchmal hilft ein Einschlafritual, das sowohl am Abend als auch am Morgen nach einer Nachtschicht funktioniert: Lesen, Tagebuch schreiben, Spaziergänge oder Entspannungsübungen. Das Ziel ist, sich bewusst zu entpflichten und den Tag oder die Nachschicht abzuschließen. Das Nickerchen vorm Fernsehgerät ist kontraproduktiv, denn es führt nur dazu, dass der Schlafdruck schon vor dem Zubettgehen abgebaut wird.

Manche gestalten auch ihr Schlafzimmer zur Wohlfühloase um - ohne Elektronikgeräte oder Arbeitsunterlagen auf dem Nachttisch. Oder sie verbannen den Wecker aus dem Blickfeld. Denn zu wissen, dass man schon bald wieder aufstehen muss, hemmt das Wiedereinschlafen in der Nacht. Techniken wie Autogenes Training oder Progressive Muskelrelaxation können das nächtliche Gedankenkarussell stoppen. Je nach Schicht und Schlafenszeit können die Maßnahmen variieren.

\footnotetext{
_ Was, wenn das alles nicht hilft?

Weeß: Bei längeren Wachphasen empfehle $i c h$, das Bett zu verlassen, denn dieses sollte nur mit Schlaf assoziiert werden. Falls Schlafmittel nötig sind, dann nur sehr leichte, kurzwirksame Präparate kurz vor dem Zubettgehen nehmen - auf Dauer helfen diese nicht. Bei einer schwerwiegenden Insomnie kann eine ambulante oder stationäre Schlaftherapie helfen. Wer dauerhaft Probleme mit dem Schichtsystem hat, sollte versuchen, in einen
}

kontinuierlichen Dienst, also in eine Dauerfrüh-oder Dauerspätschicht zu wechseln.

\begin{abstract}
_ Was können Arbeitgeber für Beschäftigte tun, um Schlafproblemen vorzubeugen? Weeß: Ideal sind flexible Schichtpläne, die den Chronotypen gerecht werden und Ausnahmen von der Regel zulassen. Möglichst wenig Nachtdienste am Stück und ausreichend Ruhezeiten zwischen den Schichten begünstigen das Schlafverhalten ebenso wie schnell- und vorwärtsrotierende Schichten. Jeweils zwei Früh-, Spät- und Nachschichten nacheinander bedeuten weniger Adaptionsstress.

Im Rahmen des betrieblichen Gesundheitsmanagements können Mitarbeitende zum Umgang mit Schichtarbeit und schlaffördernde Verhaltensweisen geschult werden. Tatsächlich beobachten wir bei Arbeitgebern ein steigendes Interesse an entsprechenden Inhouseoder Online-Seminaren - gutem Schlaf wird also immer mehr Bedeutung zugemessen.
\end{abstract}

_ Können Sie uns in Ihrer Funktion als Vorstandsmitglied der Deutschen Gesellschaft für Schlafforschung und Schlafmedizin (DGSM) weitere, wichtige Themen der Schlafforschung nennen?

Weeß: Die DGSM ist eine große wissenschaftliche Fachgesellschaft, die das Wissen zur Behandlung von über 80 wissenschaftlich definierten Schlafstörungen bündelt. Darüber hinaus ist sie in der Grundlagenforschung tätig und entwickelt allgemeine Standards zur Diagnostik und Therapie. Aktuell ist "Digitalisierung" ein großes Thema: Wir entwickeln Online-Angebote zur Behandlung von Schlafstörungen und zur Schulung niedergelassener Haus- und Fachärzte. Gerade laufen erste Studien zu Corona. Wir wissen noch nicht, ob es einen biologischen Zusammenhang zwischen Covid-19 und Insomnie gibt. Doch es zeichnet sich jetzt schon ab, dass die Pandemiesituation an sich - die Sorgen um Job, Familie und Gesundheit - vermehrt zu Schlafstörungen führt.

Das Interview führte Carolin Grehl

$\triangle$ Tipps für einen guten Schlaf vor und nach den Schichten finden Sie online über das HEILBERUFE eMag auf springerpflege.de 